\title{
Utility of autologous fibrin glue and polyglycolic acid sheet for preventing delayed bleeding associated with antithrombotic therapy after gastric ESD
}

\section{(ㄷ)(우우}

\author{
Authors \\ Shu Hoteya ${ }^{1}$ \\ Institutions \\ 1 Department of Gastroenterology, Toranomon Hospital, \\ Tokyo, Japan \\ 2 Department of Transfusion medicine, Toranomon \\ Hospital, Tokyo, Japan \\ 3 Department of Surgery, Toranomon Hospital, Tokyo, \\ Japan
}

Daisuke Kikuchi' ${ }^{1}$ Toshiro lizuka ${ }^{1}$, Shigeyoshi Makino ${ }^{2}$, Junnosuke Hayasaka ${ }^{1}$, Hiroyuki Odagiri ${ }^{1}$, Yorinari Ochiai ${ }^{1}$, Yugo Suzuki ${ }^{1}$, Kosuke Nomura ${ }^{1}$, Yu Ohkura ${ }^{3}$, Yosuke Okamoto ${ }^{1}$, Masami Tanaka ${ }^{1}$, Akira Matsui ${ }^{1}$, Toshifumi Mitani ${ }^{1}$,

submitted 1.3.2019

accepted after revision 23.8.2019

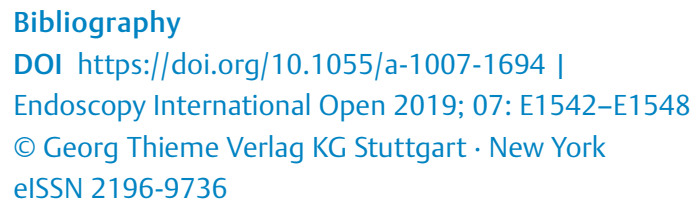

Corresponding author

Daisuke Kikuchi, MD, PhD, 2-2-2 Toranomon Minato-ku, Tokyo, Japan

Fax: $+81-3-3582-7068$

dkiku1230@gmail.com

\section{ABSTRACT}

Background and study aims Delayed bleeding is one of the most serious adverse events of gastric endoscopic submucosal dissection (ESD), especially in patients taking antithrombotic therapy. This study aimed to evaluate the utility and safety of a shielding method with autologous fibrin glue and polyglycolic acid (PGA) sheets for patients undergoing gastric ESD who are receiving antithrombotic therapy.

Patients and methods One hundred twenty-three patients who were treated with gastric ESD while receiving antithrombotic therapy between December 2014 and September 2017 were enrolled in this study. Patients who received the shielding method were classified into the shielding group.Others were classified into the conventional group. Various clinico-pathological factors were retrospectively compared between the two groups.

Results The shielding group consisted of 38 patients, and the conventional group consisted of the remaining 85 patients. In the shielding group, the rate of continuation of antithrombotic therapy was significantly higher $(68.4 \%$ vs $41.2 \%)$. Incidence of delayed bleeding was lower in the shielding group $(2.6 \%, 1 / 38)$ than in the conventional group $(14.1 \%, 12 / 85)$. In the propensity score-adjusted logistic regression analysis, the delayed bleeding rate in the shielding group tended to be lower than in the conventional group $(P=0.070)$. Allogeneic transfusion was performed in eight patients $(8 / 85,9.4 \%)$ in the conventional group and none in the shielding group $(P=0.047)$. No adverse event associated with endoscopic shielding were observed in the shielding group.

Conclusions This study suggests that a shielding method with autologous fibrin glue and PGA sheet effectively prevents delayed bleeding after gastric ESD in patients receiving antithrombotic therapy.

\section{Introduction}

Endoscopic submucosal dissection (ESD) has been a standard treatment for early gastric cancer without risk of lymph node metastasis. With technical advances in gastrointestinal endoscopy, many gastric cancer lesions are detected and treated endoscopically. The frequency and prevalence of cardiovascular disease, such as cerebral infarction and ischemic heart disease, have been increasing because of population aging and poor dietary habits. Antithrombotic therapy has thus become widely used in treating and preventing such diseases in recent years [1]. Against this background, the number of patients receiving antithrombotic therapy and requiring ESD has also been increasing.

The Japan Gastroenterological Endoscopy Society revised its guidelines for management of patients receiving antithrombo- 
tic therapy [2]. The new guidelines acknowledge the increased risk of thromboembolism on cessation of antithrombotic therapy. Following this revision, the number of gastric ESD cases with continuation of antithrombotic therapy has been increasing and the rate of delayed bleeding also is expected to increase. Therefore, new safety measures to prevent delayed bleeding are required [3].

Shielding methods with autologous fibrin glue and polyglycolic acid (PGA) sheets has been used to prevent bleeding and leakage in various surgical fields $[4,5]$. We performed autologous blood collection before gastric ESD in patients with high risk of delayed bleeding and used autologous fibrin glue and PGA sheet to cover the ESD ulcer. Herein, we evaluated the utility of this shielding method using autologous fibrin glue and PGA sheet to prevent delayed bleeding after gastric ESD in patients receiving antithrombotic therapy.

\section{Patients and methods}

\section{Patients}

A total of 903 lesions (665 patients) were treated using ESD at our hospital between December 2014 and September 2017. Of these, 123 patients (18.5\%) receiving some form of antithrombotic therapy were enrolled in this study.

We performed the shielding method with autologous fibrin glue and PGA sheet in 38 patients (Shielding group). In 85 patients, we did not perform the shielding method (Conventional group). The two groups were compared retrospectively with respect to various clinical outcomes. This study was approved by ethical committee of our hospital. In this retrospective study, only anonymous data were used, so provision of informed consent was waived.

\section{Autologous blood collection}

Autologous blood collection was performed after obtaining informed consent. At least 7 days before the scheduled ESD, $400 \mathrm{~mL}$ of blood was collected. Autologous fibrinogen was prepared manually immediately after blood collection. CryoSeal fibrin sealant was used from patient number 36 onward. Autologous fibrinogen and autologous thrombin were then prepared from the autologous blood simultaneously using CryoSeal fibrin sealant.

Patients with anemia (hemoglobin $\leq 11 \mathrm{~g} / \mathrm{dL}$ ), active infection, severe dehydration, or severe aortic stenosis were excluded. Autologous blood was stored in a freezer or refrigerator until the managing physician determined that the patient was no longer at risk of delayed bleeding.

\section{ESD procedure}

All patients in this study underwent conventional ESD performed by skilled endoscopists who had experience with more than 300 cases of gastric ESD or by endoscopists in training who were also allowed to perform ESD under the supervision of the skilled endoscopists. ESD was performed using the GIF 260 J or 2TQ260M endoscope (Olympus Optical, Tokyo, Japan). ESD was started with a Dual knife (Olympus, Tokyo, Japan) in all patients, and an additional knife, such as a Hook knife, was used

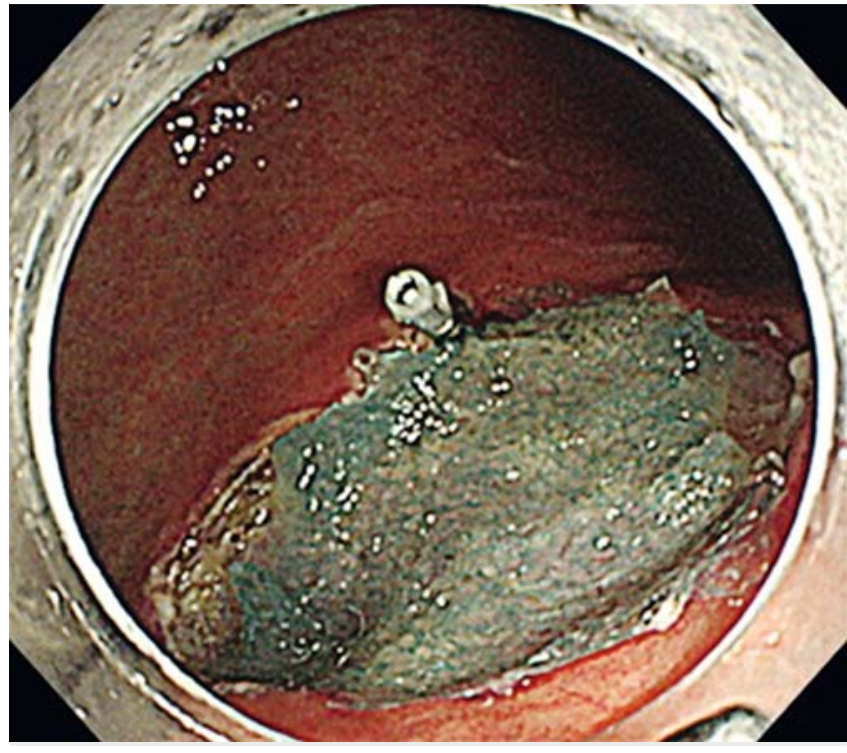

- Fig. 1 Endoscopic image of ESD ulcer with polyglicolic acid (PGA) sheet. After coagulation of visible vessel, PGA sheet was applied using biopsy forceps and fixed using clip.

when needed by the operator. A high-frequency electrosurgical unit, ICC 200 or VIO 300 D (Erbe Elektromedizin, Tubingen, Germany) was used for high-frequency current generation in this study.

After chromoendoscopy or narrow-band imaging magnification, some markings were placed around the lesion. Next, mucosal incisions were made after local injection of Glyceol (Chugai Pharmaceutical Co., Ltd., Tokyo, Japan), and submucosal dissection was performed after additional injection; the lesion was removed en bloc. After resection, the blood vessels in the ESD ulcer bed were coagulated using hemostatic forceps (Pentax Medical, Tokyo, Japan).

The decision to continue, discontinue, or switch to alternative antithrombotic therapy was made by the managing physician and specialists in cardiovascular and cerebrovascular disease based on the patient's condition. In all cases, cardiovascular or cerebrovascular specialists were consulted.

\section{Shielding method}

Shielding was performed by two skilled endoscopists (D.K. and T.I.), each with experience with more than 500 ESD. A PGA sheet was cut to the size of the ESD ulcer and immersed in autologous fibrinogen and then applied using biopsy forceps and fixed with clips ( $>$ Fig.1). All PGA sheets were attached to the ESD ulcer by the clip and pull method proposed by Ono et al. In all procedures, Ez clip (Olympus, Tokyo, Japan) was used. Autologous fibrinogen and thrombin were sprayed simultaneously to bond the PGA sheet ( $\mathbf{F i g . 2}$ ). Bovine thrombin was used in patients 1 to 35 , and autologous thrombin was used from patient number 36 onward. 


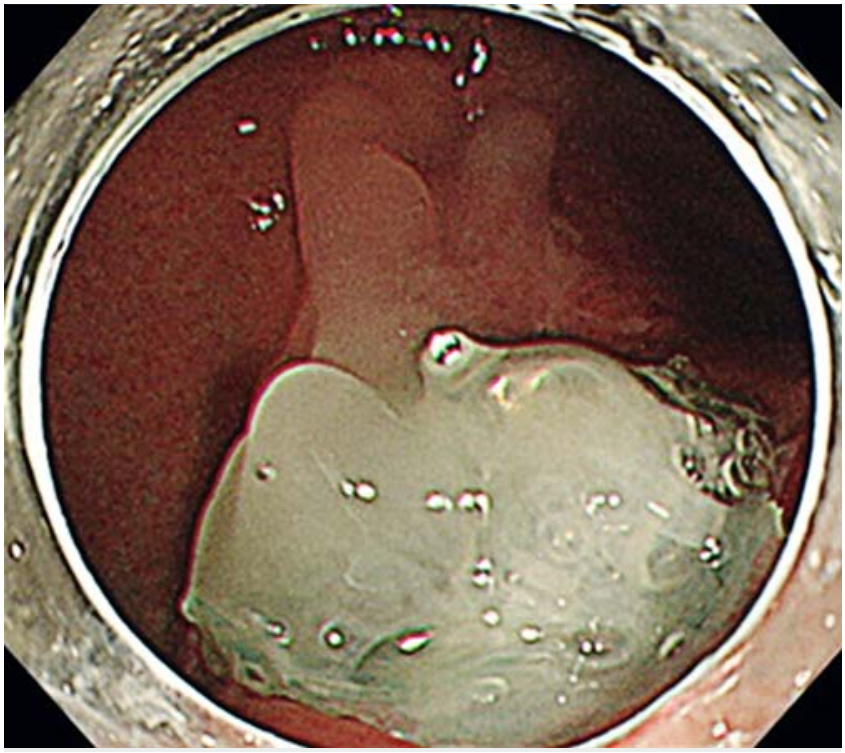

- Fig. 2 Endoscopic image of ESD ulcer after spraying autologous fibrin glue. Autologous fibrinogen and thrombin solution were sprayed simultaneously to bond the PGA sheet.

\section{Follow-up after ESD}

Post-ESD follow-up was performed according to the conventional protocol at our hospital. On the day of ESD, the patient was fasted and given intravenous fluid replacement therapy. On the day after ESD, abdominal, blood, and x-ray examinations were performed, and the managing physician decided when the patient could resume eating based on the examination results.

Then, 7 days after ESD, endoscopic examination was performed to confirm bleeding, visible blood vessels, and the status of the PGA sheet and autologous fibrin glue ( $\mathbf{F i g} . \mathbf{3}$ ). The managing physician then decided whether transfusion was needed for delayed bleeding. Blood transfusion was performed if the hemoglobin level was decreasing $<8 \mathrm{~g} / \mathrm{dL}$, or a patient's vital signs were abnormal. In the shielding group, autologous blood was scheduled to be used first and if insufficient, allogenic blood was scheduled to be used next. Proton pump inhibitor was prescribed in all cases for 8 weeks. By 8 weeks after ESD, endoscopic examination was performed to confirm scar formation at the site of the ESD ulcer.

\section{Definitions}

Delayed bleeding was defined as hematemesis, melena, other bleeding-related symptoms, or anemia (defined as a decrease in hemoglobin of $\geq 2 \mathrm{~g} / \mathrm{dL}$ compared with preoperative levels) that warranted emergency endoscopy for hemostasis. Endoscopic hemostasis for visible vessels or oozing without clinical symptoms of bleeding on second-look endoscopy was not included in delayed bleeding in this study.

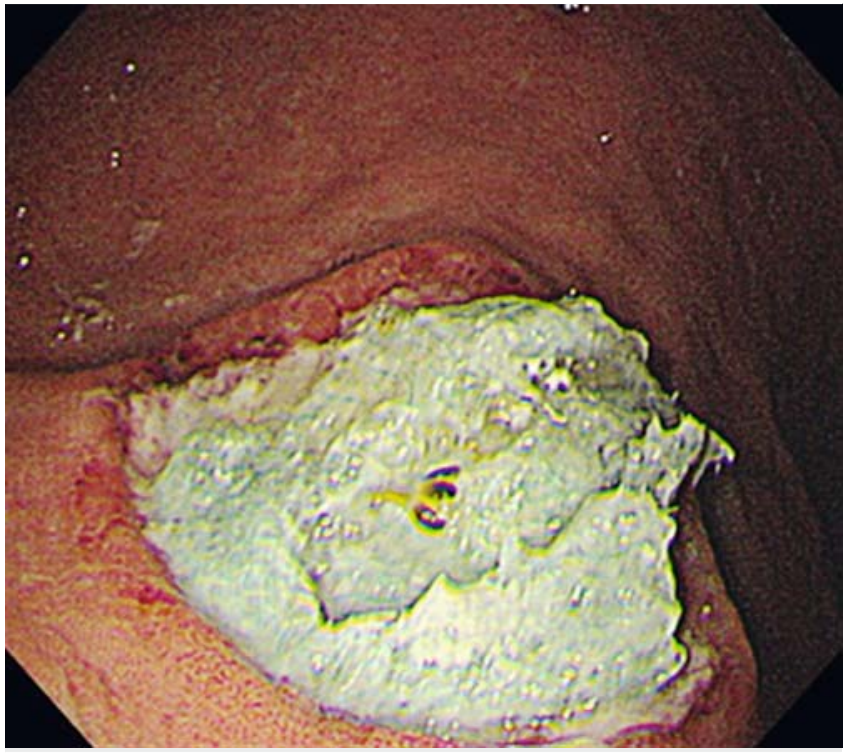

- Fig. 3 Endoscopic image of ESD ulcer 7 days after ESD. PGA sheet was recognized on the ESD ulcer.

\section{Evaluation items}

The primary endpoint was incidence of delayed bleeding. Secondary endpoints were: (1) incidence of transfusion; (2) incidence of thromboembolism; (3) incidence of serious adverse events (AEs) such as perforation or aspiration pneumonia during ESD; (4) post-ESD fasting period; (5) implementation rate of endoscopic hemostasis for post-ESD ulcer; and (6) percentage of ulcer scar formation by 8 weeks after ESD.

In the shielding group, AEs associated with shielding, incidence of autologous transfusion, and rate of attachment of the PGA sheet and autologous fibrin glue to the ESD ulcer were evaluated.

\section{Statistical analysis}

Data were analyzed using the unpaired t-test, Chi-squared test, or Mann-Whitney U-test as appropriate. $P<0.05$ was considered significant. To adjust for patient characteristics, we calculated propensity scores using a logistic regression analysis. Propensity scores were estimated using the following variables: gender, age, cerebrovascular disease, cardiovascular disease, arrythmia, diabetes mellitus, chronic renal disease on hemodialysis, lesion location, lesion size, antiplatelet therapy and anticoagulant. We calculated the C-statistics and performed logistic regression analysis to assess the association between occurrence of postESD bleeding and PGA shielding using autologous fibrin glue with adjustment for propensity scores. All statistical analyses were performed using SPSS version 20 (SPSS IBM statistics).

\section{Results}

The shielding group consisted of 38 of 123 patients (40 specimens), and the conventional group consisted of the remaining 85 patients (94 specimens). Table 1 shows patient backgrounds. Mean age was $75.6 \pm 8.2$ years in the shielding group 
- Table 1 Characteristics of patients in this study.

\begin{tabular}{|c|c|c|c|}
\hline & Shielding group & Conventional group & $P$ value \\
\hline Patients & 38 & 85 & \\
\hline Age $( \pm S D)$ & $75.6 \pm 8.2$ & $76.5 \pm 7.1$ & 0.43 \\
\hline Gender (male/female) & $31 / 7$ & $74 / 11$ & 0.30 \\
\hline \multicolumn{4}{|l|}{ Comorbidity } \\
\hline - Cerebrovascular disease(\%, n/n) & $31.5(12 / 38)$ & $23.5(20 / 85)$ & 0.24 \\
\hline - Cardiovascular disease (\%, n/n) & $36.8(14 / 38)$ & $38.8(33 / 85)$ & 0.50 \\
\hline " Arrhythmia (\%, n/n) & $31.5(12 / 38)$ & $35.2(30 / 85)$ & 0.43 \\
\hline - Diabetes mellitus (\%, n/n) & $15.8(6 / 38)$ & $29.4(25 / 85)$ & 0.081 \\
\hline - Hemodialysis (\%, n/n) & $2.6(1 / 38)$ & $4.7(4 / 85)$ & 0.42 \\
\hline
\end{tabular}

- Table 2 Status of antithrombotic therapy in this study.

\begin{tabular}{|c|c|c|c|}
\hline & Shielding group & Conventional group & $P$ value \\
\hline Continuation rate $(\%, \mathrm{n} / \mathrm{n})$ & $68.4(26 / 38)$ & $41.1(35 / 85)$ & 0.004 \\
\hline Multiple medication $(\%, \mathrm{n} / \mathrm{n})$ & $23.7(9 / 38)$ & $16.5(14 / 85)$ & 0.24 \\
\hline Anticoagulant (\%, $\mathrm{n} / \mathrm{n})$ & $36.8(14 / 38)$ & $34.1(29 / 85)$ & 0.46 \\
\hline Heparin displacement $(\%, n / n)$ & $15.8(6 / 38)$ & $11.8(11 / 85)$ & 0.43 \\
\hline Type of antiplatelet therapy $(\%, n / n)$ & & & 0.48 \\
\hline - Aspirin & $39.5(15 / 38)$ & $44.7(38 / 85)$ & \\
\hline - Clopidogrel & $15.8(6 / 38)$ & $9.4(8 / 85)$ & \\
\hline - Cilostazol & $13.2(5 / 38)$ & $9.4(8 / 85)$ & \\
\hline - Ticlopidine & $5.2(2 / 38)$ & $2.4(2 / 85)$ & \\
\hline - Others & $15.8(6 / 38)$ & $20.0(17 / 85)$ & \\
\hline
\end{tabular}

Table 3 Characteristics of lesions in this study.

\begin{tabular}{|l|l|l|l|}
\hline & Shielding group & Conventional group & P value \\
\hline No. of Lesions & 45 & 106 & $16.6 \pm 13.3$ \\
\hline - Tumor size $(\mathrm{mm} \pm \mathrm{SD})$ & $12.6 \pm 9.2$ & $88 / 18$ & 0.11 \\
\hline - Tumor depth $(\mathrm{m} / \mathrm{sm})$ & $42 / 3$ & $19 / 32 / 55$ & 0.070 \\
\hline - Lesion location $(\mathrm{U} / \mathrm{M} / \mathrm{L})$ & $5 / 16 / 24$ & 0.61 \\
\hline
\end{tabular}

and $76.5 \pm 7.1$ years in the conventional group, with no significant intergroup difference $(P=0.43)$. Comorbid diseases were cerebrovascular disease $(12 / 38,31.5 \%)$, cardiovascular disease $(14 / 38,36.8 \%)$, chronic renal disease on hemodialysis $(1 / 38$, $2.6 \%)$, and arrhythmia $(12 / 38,31.5 \%)$ in the shielding group and cerebrovascular disease $(20 / 85,23.5 \%)$, cardiovascular disease $(33 / 85,38.8 \%)$, chronic renal disease on hemodialysis $(4 / 85,4.7 \%)$, and arrhythmia $(30 / 85,35.3 \%)$ in the conventional group (N.S.). Detailed information on antithrombotic therapy in this study is shown in - Table 2. Rates of anticoagulation therapy, use of multiple medications, and heparin displacement were $36.8 \%(14 / 38), 23.7 \%(9 / 38)$, and $15.8 \%(6 / 38)$ in the shielding group and 34.1\% (29/85), 16.5\% (14/85), and $12.9 \%(11 / 85)$ in the conventional group (N.S.), respectively. In particular, both groups were equivalent regarding the content of antiplatelet therapy. The rate of continuation of antithrombotic therapy was $68.4 \%(26 / 38)$ in the shielding group and $41.2 \%(35 / 85)$ in the conventional group, with a significantly higher rate in the former $(P=0.004)$.

Lesion characteristics are shown in $>$ Table 3 . In the shielding group, 45 lesions were resected from 38 patients. Mean tumor size was $12.6 \pm 9.2 \mathrm{~mm}$. Of the 45 lesions, 42 were intramucosal lesions and three were submucosal invasive carcinomas. In the conventional group, 106 lesions were resected from 85 
- Table4 Proportion of clinical outcomes following ESD in the shielding and conventional groups.

\begin{tabular}{|l|l|l|}
\hline & Shielding group & \multicolumn{1}{l|}{ Conventional group } \\
\hline Patients & 38 & 85 \\
\hline Delayed bleeding $(\%, \mathrm{n} / \mathrm{n})$ & $2.6(1 / 38)$ & $14.1(12 / 85)$ \\
\hline Blood transfusion $(\%, \mathrm{n} / \mathrm{n})$ & $0(0 / 38)$ & $9.4(8 / 85)$ \\
\hline Thromboembolism $(\%, \mathrm{n} / \mathrm{n})$ & $0(0 / 38)$ & $0(0.85)$ \\
\hline Perforation $(\%, \mathrm{n} / \mathrm{n})$ & $0(0 / 38)$ & $1.2(1 / 85)$ \\
\hline Pneumonia $(\%, \mathrm{n} / \mathrm{n})$ & $0(0 / 38)$ & $0(0 / 85)$ \\
\hline Endoscopic hemostasis $(\%, \mathrm{n} / \mathrm{n})$ & $15.8(6 / 38)$ & $41.2(35 / 85)$ \\
\hline Scar formation 8 weeks after ESD $(\%, \mathrm{n} / \mathrm{n})$ & $80.0(32 / 40)$ & $85.9(67 / 78)$ \\
\hline ESD, endoscopic submucosal dissection & & 0.047 \\
\hline
\end{tabular}

- Table 5 Propensity score-adjusted logistic regression analysis for delayed bleeding.

\begin{tabular}{|l|l|l|l|}
\hline & Odds ratio & $95 \% \mathbf{C l}$ & $P$ value \\
\hline Shielding group & 0.132 & $0.015-1.179$ & 0.070 \\
\hline
\end{tabular}

patients. Mean tumor size was $16.6 \pm 13.3 \mathrm{~mm}$. Of the $106 \mathrm{le}-$ sions, 88 were intramucosal lesions and 18 were submucosal invasive carcinomas. Mean tumor size did not differ significantly between the groups $(P=0.11)$.

The results of this study are shown in $\triangleright$ Table 4 and $\triangleright$ Table5. Incidence of delayed bleeding, the primary endpoint of this study, was lower in the shielding group $(2.6 \%, 1 / 38)$ than in the conventional group $(14.1 \%, 12 / 85)$. C statistic of propensity score in this study was 0.679 . In the propensity score-adjusted logistic regression analysis, the odds ratio for delayed bleeding in the shielding group was $0.132(95 \% \mathrm{Cl}, 0.015-$ $1.179, P=0.070)$. The delayed bleeding rate in the shielding group was not significant but tended to be lower than that in the conventional group.

In the conventional group, delayed bleeding occurred 13 times in 12 patients, with a mean duration of $6.8 \pm 6.7$ days between ESD and the first delayed bleeding. In the shielding group, one patient had melena on post-ESD Day 9, as a sign of delayed bleeding. Blood transfusion was performed in eight patients $(8 / 85,9.4 \%)$ in the conventional group and none in the shielding group, with a significant intergroup difference $(P=$ 0.047). Thromboembolism was not observed in either group. One case of perforation was observed in the conventional group. None of the patients in the shielding group developed pneumonia or fever above $38^{\circ} \mathrm{C}$. In the conventional group, although none had pneumonia, four patients developed fever above $38^{\circ} \mathrm{C}$ after ESD. They were treated with antibiotics and had favorable outcomes. Hospital meals started $1.5 \pm 0.7$ days after ESD in the shielding group and $1.8 \pm 1.4$ days in the conventional group (N.S.). In an 8-week period after ESD, endoscopic hemostasis including preventive hemostasis was performed in $15.8 \%$ patients $(6 / 38)$ in the shielding group and
$41.2 \%$ of patients $(35 / 85)$ in the conventional group, with a significant intergroup difference $(P=0.017)$. The rate of scar formation in the same 8 -week period was $80 \%(32 / 40)$ in the shielding group and $85.9 \%(67 / 78)$ in the conventional group $(P=0.31)$.

In the shielding group, all ESD ulcer were covered with PGA sheets and fibrin glue just after the ESD procedure. No AEs associated with endoscopic shielding were observed. The patient with delayed bleeding in the shielding group had anemia, but it was mild, so no autologous transfusion was performed. Endoscopy performed at 1 week after ESD revealed that $75 \%$ (30/40) of PGA sheets remained attached.

\section{Discussion}

Bleeding is a major problem in gastric ESD and is largely classified into intraoperative bleeding and delayed bleeding. Incidence of both types of bleeding is high, with potentially severe consequences, in gastric ESD compared with pharyngeal, esophageal, and colorectal ESD. Intraoperative bleeding has been progressively addressed through improvement in endoscopic skills and development of new instruments [6,7], while delayed bleeding remains unresolved. Incidence of delayed bleeding after gastric ESD varies among studies, ranging from $4 \%$ to $10 \%$. Risk factors for delayed bleeding include lesions in the $L$ region of the stomach, large resection specimen, cirrhosis, dialysis, and antithrombotic therapy [8-10]. Antithrombotic therapy is a major problem because of its widespread use in recent years. As a novel strategy to overcome this problem, we perform endoscopic shielding with autologous fibrin glue and PGA sheets [4].

Various preventive measures for delayed bleeding are attempted after ESD, one of which is administration of proton pump inhibitors (PPIs) [11]. Many institutions prescribe PPIs after gastric ESD to prevent delayed bleeding because the efficacy was shown to be superior to that of histamine $\mathrm{H} 2$-receptor antagonists [12]. We also administered PPIs to all patients in this study. Previous research has also shown that the recently commercialized vonoprazan facilitates healing of post-ESD ulcer [13]. It is anticipated that further research will verify the as- 
sociation between vonoprazan and delayed bleeding. There are a few reports about the utility of a shielding method for delayed bleeding after gastric ESD [14-16]. However, in these reports, fibrin glue was prepared from donor, not autologous, blood products. The safety of the fibrin glue is thought to be high, but because it is nonautologous, the possibility of infection or allergic reaction cannot be excluded completely. In the current study, such risk is virtually nonexistent due to use of autologous fibrin glue. Also, collection of autologous blood enables us to avert allogeneic transfusion at the time of bleeding. In the current study, eight patients underwent allogeneic transfusion in the conventional group, but post-ESD allogeneic transfusion may be avoided in the future by further incorporating the safety measures used in this study. This safety measure may lead to improvement in safety in gastric ESD in patients with high risk of delayed bleeding.

Endoscopic shielding with fibrin glue and PGA sheet has been performed in various regions of the digestive tract, such as the duodenum, stomach, colon, and esophagus [14-20]. The purpose of shielding varies among organs. Prevention and treatment of perforation is the primary purpose in the duodenum and colon. In the esophagus, however, endoscopic shielding is mainly performed to prevent stricture because previous research has shown the prevention of scar contracture by PGA sheet [21], promoting its application in stricture. Indeed, in our study, the rate of scar formation at 2 months after ESD was lower, albeit insignificant, in the shielding group, suggesting the suppression of scar formation by the PGA sheet. In the stomach, the sheet is normally used to prevent delayed bleeding $[14,15]$, and its utility has been reported previously. However, a multicenter, randomized, controlled trial (RCT) could not demonstrate the utility of this method for patients at high risk of delayed bleeding [16]. In this trial, high-risk patients were defined as those with regular intake of antithrombotic agents or those expected to undergo large mucosal resection (>40 mm). Fewer than $40 \%$ of the patients in this trial were taking antithrombotic therapy, and most of them were taking only aspirin. In the next RCT, higher-risk patients such as those receiving dual antiplatelet or anticoagulant therapy should be enrolled. In our study, about half of the patients were continuing antithrombotic therapy during ESD, and over $30 \%$ of the patients were taking an anticoagulant. And we could observe a lower incidence of delayed bleeding in the shielding group, which suggests that the shielding method may be useful for preventing delayed bleeding for patients receiving antithrombotic therapy, and the efficacy of autologous fibrin glue is comparable to that of nonautologous fibrin glue. However, there are no data comparing autologous and non-autologous fibrin glue, so a prospective comparable study should be scheduled in the future.

This study has a few limitations. First is the method used to apply the PGA sheet to the ulcer bed. We used the clip-and-pull method proposed by Ono et al. [22], in which PGA sheets are stabilized by clips. Clips, however, fall off when the PGA sheets are peeling away, raising the possibility of bleeding from the site. It takes skill, experience, and time to effectively apply the PGA sheet over the ESD ulcer. In this study, endoscopic shielding was performed by two skilled endoscopists. It should be kept in mind that the utility of the shielding method may decrease when the procedure is performed by novice endoscopists. Second, hemostasis may be difficult if delayed bleeding occurs beneath the PGA sheet. In our study, only one patient had delayed bleeding after endoscopic shielding, but hemostasis was achieved without any problem because the PGA sheet had been peeled off before delayed bleeding began. We plan to accumulate more cases to address this issue. Third, this was a retrospective single-center study. Specifically, lesion size was larger in the conventional group and this might have affected incidence of delayed bleeding. In the future, a large-scale multicenter study is needed to verify the utility of the shielding method used in this study.

\section{Conclusion}

In conclusion, the results of this study suggest that the endoscopic shielding method with autologous fibrin glue and PGA sheet effectively prevents delayed bleeding after gastric ESD in patients receiving antithrombotic therapy. The results of this study warrant validation in a prospective study.

\section{Competing interests}

None

References

[1] Application of U.S. guidelines in other contries: aspirin for the primary prevention of prevention of cardiovascular events in Japan. Am J Med 2014; 117: 459-468

[2] Fujimoto K, Fujishiro M, Kato M et al. Guidelines for gastroenterological endoscopy in patients undergoing antithrombotic treatment. Dig Endosc 2014; 26: 1 - 14

[3] Kikuchi D, lizuka T, Makino S et al. Feasibility of autologous fibrin glue and polyglycolic acid sheets to prevent delayed bleeding after endoscopic submucosal dissection of gastric neoplasms in patients receiving antithrombotic therapy. Gastroenterol Res Pract 2018; 2018: 2174957

[4] Yano T, Haro A, Shikada Y et al. A unique method for repairing intraoperative pulmonary air leakage with both polyglycolic acid sheets and fibrin glue. World J Surg 2012; 36: $463-467$

[5] Kouketsu A, Nogami S, Fujiwara M et al. Clinical evaluations of autologous fibrin glue and polyglycolic acid sheets as oral surgical wound coverings after partial glossectomy. J Craniomaxillofac Surg 2016; 44: 964-968

[6] Kikuchi D, lizuka T, Hoteya S et al. Usefulness of endoscopic ultrasound for the prediction of intraoperative bleeding of endoscopic submucosal dissection for gastric neoplasms. J Gastroenterol Hepatol 2011; 26: $68-72$

[7] Kikuchi D, lizuka T, Hoteya $S$ et al. Prospective study about the utility of endoscopic ultrasound for predicting the safety of endoscopic submucosal dissection in early gastric cancer (T-HOPE 0801). Gastroenterol Res Pract 2013; 2013: 329385

[8] Furuhata T, Kaise M, Hoteya $S$ et al. Postoperative bleeding after gastric endoscopic submucosal dissection in patients receiving antithrombotic therapy. Gastric Cancer 2017; 20: 207-214 
[9] Oda I, Suzuki H, Nonaka S et al. Complications of gastric endoscopic submucosal dissection. Dig Endosc 2013; 25: $71-78$

[10] Ueki N, Futagami S, Akimoto T et al. Effect of antithrombotic therapy and long endoscopic submucosal dissection procedure time on early and delayed postoperative bleeding. Digestion 2017; 96: 21-28

[11] Niimi K, Fujishiro M, Goto O et al. Prospective single-arm trial of twoweek rabeprazole treatment for ulcer healing after gastric endoscopic submucosal dissection. Dig Endosc 2012; 24: 110-116

[12] Yang Z, Wu Q, Liu Z et al. Proton pump inhibitors versus histamine-2receptor antagonists for the management of iatrogenic gastric ulcer after endoscopic mucosal resection or endoscopic submucosal dissection: a meta-analysis of randomized trials. Digestion 2011; 84: $315-320$

[13] Maruoka D, Arai M, Kasamatsu S et al. Vonoprazan is superior to proton pump inhibitors in healing artificial ulcers of the stomach postendoscopic submucosal dissection: A propensity score-matching analysis. Dig Endosc 2017; 29: 57-64

[14] Tsuji Y, Fujishiro M, Kodashima S et al. Polyglycolic acid sheets and fibrin glue decrease the risk of bleeding after endoscopic submucosal dissection of gastric neoplasms (with video). Gastrointest Endosc 2015; 81: 906 - 912

[15] Kawata N, Ono H, Takizawa K et al. Efficacy of polyglycolic acid sheets and fibrin glue for prevention of bleeding after gastric endoscopic submucosal dissection in patients under continued antithrombotic agents. Gastric Cancer 2018; 2: 696-702
[16] Kataoka Y, Tsuji Y, Hirasawa K et al. Endoscopic tissue shielding to prevent bleeding after endoscopic submucosal dissection: a prospective multicenter randomized controlled trial. Endoscopy 2019; 51: $619-627$

[17] Takimoto K, Toyonaga T, Matsuyama K. Endoscopic tissue shielding to prevent delayed perforation associated with endoscopic submucosal dissection for duodenal neoplasms. Endoscopy 2012; 44: E414 - 415

[18] lizuka T, Kikuchi D, Yamada A et al. Polyglycolic acid sheet application to prevent esophageal stricture after endoscopic submucosal dissection for esophageal squamous cell carcinoma. Endoscopy 2015; 47: $341-344$

[19] Sakaguchi Y, Tsuji Y, Ono S et al. Polyglycolic acid sheets with fibrin glue can prevent esophageal stricture after endoscopic submucosal dissection. Endoscopy 2015; 47: $336-340$

[20] Tsuji Y, Ohata K, Gunji T et al. Endoscopic tissue shielding method with polyglycolic acid sheets and fibrin glue to cover wounds after colorectal endoscopic submucosal dissection (with video). Gastrointest Endosc 2014; 79: 151 - 155

[21] Takeuchi J, Suzuki H, Murata M et al. Clinical evaluation of application of polyglycolic acid sheet and fibrin glue spray for partial glossectomy. J Oral Maxillofac Surg 2013; 71: e126-e131

[22] Ono S, Tsuji Y, Fujishiro M et al. An effective technique for delivery of polyglycolic acid sheet after endoscopic submucosal dissection of the esophagus: the clip and pull method. Endoscopy 2014; 46: E44 - E45 